\title{
EXISTENCE AND UNIQUENESS OF THE SOLUTIONS TO IMPULSIVE NONLINEAR INTEGRO-DIFFERENTIAL EQUATIONS WITH NONLOCAL BOUNDARY CONDITIONS
}

\author{
MISIR J. MARDANOV, YAGUB A. SHARIFOV, AND FARAH M. ZEYNALLI
}

\begin{abstract}
In the paper a system of ordinary impulsive integro-differential equations with nonlocal conditions is studied. At first the boundary value problem is reduced to the equivalent integral equation. Then, using the theorem on fixed points, the condition on the existence and uniqueness of the solution of the boundary value problem is obtained. Continuous dependence of the solutions on the right hand side of boundary conditions is also set up.
\end{abstract}

\section{Introduction}

A lot of problems of physics, engineering, biology and economy are described by differential and integro-differential equations whose solutions are the functions with first kind discontinuities at fixed and unfixed moments of time. Such differential equations were studied rather well in $[6,8,9,12,13,15-17]$ and they were called impulsive differential equations. In the above mentioned papers, mainly the differential equations with local conditions are studied. However, the last years there is a great interest to impulsive differential and integro-differential equations with nonlocal boundary conditions, by which a number of practical processes are described.

Today, there exist a great number of works devoted to ordinary impulsive differential and integro-differential equations with nonlocal boundary conditions in which the theorem on the existence of solutions are proved for different types of nonlocal conditions [3-8,11,13,18-22].

Integral boundary conditions have applications in numerous fields such as modeling and analyzing of many physical systems including blood flow problems, chemical engineering, thermoelasticity, underground water flow, population dynamics and etc. For more details of integral boundary conditions, see $[1,13,14]$ and references therein.

Note that numerical methods for multipoint and integral boundary problems for first-order ordinary differential equations were developed in $[1,2]$.

2010 Mathematics Subject Classification. 34A37, 34B37, 34B10.

Key words and phrases. impulsive differential equations, nonlocal boundary conditions, existence and uniqueness of solutions, continuous dependence of solutions. 
In the present paper we study a nonlocal boundary value problem for the system of impulsive ordinary integro-differential equations whose boundary conditions include pointwise and integral terms. It should be noted that the boundary value problem under consideration is rather general. In special cases it covers the Cauchy problem and "pure" integral condition. The existence and uniqueness of the solution of the boundary value problem and also continuous dependence of the solution on the right hand side of boundary conditions, is studied.

\section{Problem statement}

We study existence and uniqueness of the solution of the system of integrodifferential equations

$$
\dot{x}(t)=f\left(t, x(t), \int_{0}^{t} g(t, s, x(s)) d s\right), t \in[0, T], t \neq t_{i}, i=1,2, \ldots, p,
$$

with nonlocal boundary conditions

$$
A x(0)+\int_{0}^{T} n(t) x(t) d t=B,
$$

under the impulse actions

$$
x\left(t_{i}^{+}\right)-x\left(t_{i}\right)=I_{i}\left(x\left(t_{i}\right)\right), \quad i=1,2, \ldots, p,
$$

where $0=t_{0}<t_{1}<\ldots<t_{p}<t_{p+1}=T, A \in R^{n \times n}, n(t) \in R^{n \times n}$ are the given matrices, and det $N \neq 0, N=A+\int_{0}^{T} n(t) d t ; f:[0, T] \times R^{n} \rightarrow R^{n}, g:[0, T] \times$ $[0, T] \times R^{n} \rightarrow R^{n}, I_{i}: R^{n} \rightarrow R^{n}$ are the given functions; $\Delta x\left(t_{i}\right)=x\left(t_{i}^{+}\right)-x\left(t_{i}^{-}\right)$, where $x\left(t_{i}^{+}\right)=\lim _{h \rightarrow 0^{+}} x\left(t_{i}+h\right), x\left(t_{i}^{-}\right)=\lim _{h \rightarrow 0^{+}} x\left(t_{i}-h\right)=x\left(t_{i}\right)$ are the right and left hand limits of the function $x(t)$ at the point $t=t_{i}$, respectively.

\section{Auxiliary facts}

We give some definitions and auxiliary facts that will be used in the sequel. By $C\left([0, T]: R^{n}\right)$ we will denote the Banach space consisting of continuous vector functions $x(t)$ determined on the interval $[0, T]$, with the values in $R^{n}$ and with the norm $\|x\|=\max _{[0, T]}|x(t)|$, where $|\cdot|$ denotes the norm in $R^{n}$.

By $P C\left([0, T], R^{n}\right)$ we denote the linear space

$$
P C\left([0, T], R^{n}\right)=\left\{x:[0, T] \rightarrow R^{n} ; x(t) \in C\left(\left(t_{i}, t_{i+1}\right], R^{n}\right),\right.
$$

$i=0,1, \ldots, p ; x\left(t_{i}^{+}\right)$and $x\left(t_{i}^{-}\right), i=1,2, \ldots, p$ exist and are finite; $\left.x\left(t_{i}^{-}\right)=x\left(t_{i}\right)\right\}$.

Obviously, the linear space $P C\left([0, T] ; R^{n}\right)$ is Banach with the norm

$$
\|x\|_{P c}=\max \left\{\|x\|_{C\left(\left(t_{i}, t_{i+1}\right]\right)}, i=0,1, \ldots, p\right\} .
$$

We determine the solution of the boundary value problem (2.1)-(2.3) in the following way. 
Definition 3.1. The function $x(t) \in P C\left([0, T]: R^{n}\right)$ is said to be the solution of boundary value problem (2.1) - (2.3) if for any $t \in[0, T], t \neq t_{i}, \quad i=1,2, \ldots, p$,

$$
\dot{x}(t)=f\left(t, x(t), \int_{0}^{t} g(t, s, x(s)) d s\right)
$$

and for $t=t_{i} i=1,2, \ldots, p 0<t_{1}<t_{2}<\ldots<t_{p}<T$

$$
\Delta x\left(t_{i}\right)=x\left(t_{i}^{+}\right)-x\left(t_{i}\right)=I_{i}\left(x\left(t_{i}\right)\right) .
$$

In addition, the function $x(t)$ satisfies the boundary condition (2.2).

We introduce the following function:

$$
K(t, \tau)=\left\{\begin{array}{l}
N^{-1}\left(A+\int_{0}^{t} n(\tau) d \tau\right), \quad 0 \leq \tau \leq t \\
-N^{-1} \int_{t}^{T} n(\tau) d \tau, \quad t<\tau \leq T
\end{array}\right.
$$

Lemma 3.1. Let $y \in C\left([0, T] ; R^{n}\right) \quad a_{i} \in R^{n} i=1,2, \ldots, p$. Then the differential equation

$$
\dot{x}(t)=y(t)
$$

with impulse actions

$$
x\left(t_{i}^{+}\right)-x\left(t_{i}\right)=a_{i}, i=1,2, \ldots, p ; 0<t_{1}<t_{2}<\ldots<t_{p}<T,
$$

and nonlocal conditions

$$
A x(0)+\int_{0}^{T} n(t) x(t) d t=B
$$

has a unique solution $x(t) \in P C\left([0, T], R^{n}\right)$ and is expressed by the following formula

$$
x(t)=N^{-1} B+\int_{0}^{T} K(t, \tau) y(\tau) d \tau+\sum_{0<t_{i}<t} K\left(t, t_{i}\right) a_{i}
$$

for $t \in\left(t_{i}, t_{i+1}\right], i=0,1, \ldots, p$.

Proof. Let the function $x(t) \in P C\left([0, T], R^{n}\right)$ be the solution of boundary value problem (3.1) - (3.3). Then integrating equation (3.1) on the interval $t \in\left(0, t_{i+1}\right)$, we get

$$
\begin{gathered}
\int_{0}^{t} y(s) d s=\int_{0}^{t} \dot{x}(s) d s= \\
=\left[x\left(t_{1}\right)-x\left(0^{+}\right)\right]+\left[x\left(t_{2}\right)-x\left(t_{1}^{+}\right)\right]+\ldots+\left[x(t)-x\left(t_{i}{ }^{+}\right)\right]= \\
=-x(0)-\left[x\left(t_{1}^{+}\right)-x\left(t_{1}\right)\right]-\left[x\left(t_{2}{ }^{+}\right)-x\left(t_{2}\right)\right]-\ldots- \\
-\left[x\left(t_{i}{ }^{+}\right)-x\left(t_{i}\right)\right]+x(t) .
\end{gathered}
$$

Taking condition (3.2) into account in the last equality, we obtain

$$
x(t)=x(0)+\int_{0}^{t} y(s) d s+\sum_{0<t_{i}<t} a_{i} .
$$


Now we require that the function $x(t) \in P C\left([0, T], R^{n}\right)$ determined by equality (3.5) satisfy the boundary condition (3.3)

$$
\left(A+\int_{0}^{T} n(t) d t\right) x(0)=B-\int_{0}^{T} n(t) \int_{0}^{t} y(s) d s d t-\int_{0}^{T} n(t) \sum_{0<t_{i}<t} a_{i} d t .
$$

As det $N \neq 0$, from (3.6) we have

$$
x(0)=N^{-1}\left[B-\int_{0}^{T} n(t) \int_{0}^{t} y(s) d s d t-\int_{0}^{T} n(t) \sum_{0<t_{i}<t} a_{i} d t\right] .
$$

The value of $x(0)$ determined by equality (3.7), is taken into account in equality (3.5). Then

$$
\begin{gathered}
x(t)=N^{-1}\left[B-\int_{0}^{T} n(t) \int_{0}^{t} y(s) d s d t-\int_{0}^{T} n(t) \sum_{0<t_{i}<t} a_{i} d t\right]+ \\
+\int_{0}^{t} y(s) d s+\sum_{0<t_{i}<t} a_{i} .
\end{gathered}
$$

As we have the equalities

$$
\begin{aligned}
\int_{0}^{T} n(t) \int_{0}^{t} y(s) d s d t & =\int_{0}^{T} \int_{t}^{T} n(s) d s y(t) d t \\
\int_{0}^{T} n(t) \sum_{0<t_{i}<t} a_{i} d t & =\sum_{0<t_{i}<T} \int_{t_{i}}^{T} n(t) d t a_{i},
\end{aligned}
$$

then from (3.8) we get

$$
\begin{gathered}
x(t)=N^{-1} B-N^{-1} \int_{0}^{T} \int_{t}^{T} n(s) d s f(t) d t- \\
-N^{-1} \sum_{0<t_{i}<t} \int_{t_{i}}^{T} n(t) d t a_{i}+\int_{0}^{t} y(s) d s+\sum_{0<t_{i}<t} a_{i} .
\end{gathered}
$$

Here we perform some simplications. Obviously, the following equalities hold:

$$
\begin{gathered}
\int_{0}^{t} y(s) d s-N^{-1} \int_{0}^{T} \int_{t}^{T} n(s) d s y(t) d t= \\
=N^{-1} \int_{0}^{t}\left(A+\int_{0}^{\tau} n(s) d s\right) y(\tau) d \tau-N^{-1} \int_{t}^{T} \int_{\tau}^{T} n(s) d s y(\tau) d \tau,
\end{gathered}
$$




$$
\begin{gathered}
\sum_{0<t_{i}<t} a_{i}-N^{-1} \sum_{0<t_{i}<T} \int_{t_{i}}^{T} n(t) d t a_{i}= \\
=N^{-1} \sum_{0<t_{i}<t}\left(A+\int_{0}^{t_{i}} n(t) d t\right) a_{i}-\sum_{t<t_{i+1}<T} N^{-1} \int_{t_{i}}^{T} n(t) d t a_{i} .
\end{gathered}
$$

Taking into account (3.10) and (3.11) in (3.9), we get formula (3.4).

Remark. The validity of the following statements follows from formula (3.4):

(i) The constant vector-function $x(t)=N^{-1} B$ is the solution of the differential equation

$$
\dot{x}(t)=0
$$

with non-local conditions

$$
A x(0)+\int_{0}^{T} n(t) x(t) d t=B .
$$

(ii) The function $x(t)=\int_{0}^{T} K(t, s) y(s) d(s)$ is the solution of the differential equation

$$
\dot{x}(t)=y(t)
$$

with non-local condition

$$
A x(0)+\int_{0}^{T} n(t) x(t) d t=0 .
$$

Here the matrix of the function $K(t, s)$ is the Green function of the given problem.

(iii) The piecewise-constant function

$$
x(t)=\sum_{0<t_{i}<t} K\left(t, t_{i}\right) a_{k}, \quad i=1,2, \ldots, p,
$$

is the solution of the differential equation

$$
\dot{x}(t)=0
$$

with impulse actions

$$
x\left(t_{i}^{+}\right)-x\left(t_{i}\right)=a_{i}, \quad i=1,2, \ldots, p .
$$

and the boundary condition

$$
A x(0)+\int_{0}^{T} n(t) x(t) d t=0 .
$$

Lemma 3.2. Assume that $f \in C\left([0, T] \times R^{n} \times R^{n}, R^{n}\right)$ and $I_{i}(x) \in C\left(R^{n}\right)$. Then the function $x(t) \in P C\left([0, T], R^{n}\right)$ is the solution of boundary value problem 
(2.1) - (2.3) if ad only if the function $x(t) \in P C\left([0, T], R^{n}\right)$ is the solution of the integral equation with impulse actions

$$
\begin{aligned}
x(t)=N^{-1} B+\int_{0}^{T} K(t, s) f\left(s, x(s), \int_{0}^{s} g(s, \tau, x(\tau)) d \tau\right) d s+ \\
\quad+\sum_{i=1}^{P} K\left(t, t_{i}\right) I_{i}\left(x\left(t_{i}\right)\right),
\end{aligned}
$$

for $t \in\left(t_{i}, t_{i+1}\right), \quad i=0,1, \ldots, p$.

Proof. Let $x(t) \in P C\left([0, T], R^{n}\right)$ be the solution of the boundary value problem. Then similarly to lemma 1 , we can show that the function $x(t) \in P C\left([0, T], R^{n}\right)$ satisfies the integral equation (3.12).

The inverse is also true. By direct calculations we can be convinced that the solution of integral equation (3.12) satisfies equation (2.1), boundary condition (2.3) and also impulse conditions (2.2) as well.

The lemma is proved.

\section{Main results}

The first main result of this section is based on the Banach fixed point principle. The theorem on the existence and uniqueness of the solution of boundary value problem (2.1) - (2.3) was proved based on this principle.

Theorem 4.1. Assume that the following conditions are fulfilled:

(H1) There exist constants $M_{1} \geq 0$ and $M_{2} \geq 0$ such that

$$
\begin{gathered}
|f(t, x, y)-f(t, \bar{x}, \bar{y})| \leq M_{1}(|x-\bar{x}|+|y-\bar{y}|), \\
|g(t, s, x)-g(t, s, y)| \leq M_{2}|x-y|,
\end{gathered}
$$

for any $t \in[0, T]$ and for all $(x, y) \in R^{2 n}$ and $(\bar{x}, \bar{y}) \in R^{2 n}$;

(H2) There exist constants $l_{i} \geq 0, i=1,2, \ldots, p$ such that

$$
\left|I_{i}(x)-I_{i}(y)\right| \leq l_{i}|x-y|
$$

for any $x, y \in R^{n}$.

If

$$
L=S\left(M_{1} T\left(1+\frac{M_{2} T}{2}\right)+\sum_{k=1}^{P} l_{k}\right)<1,
$$

the boundary value problem (2.1) - (2.3) has a unique solution.

Here the number $S$ is determined by the equality

$$
S=\max _{0 \leq t, s \leq T}\|K(t, s)\| .
$$

Proof. For the proof we use the Banach fixed point principle.

Let us define the operator $F: P C\left([0, T] ; R^{n}\right) \rightarrow P C\left([0, T] \times R^{n}\right)$ from the relation

$$
(F x)(t)=N^{-1} B+\int_{0}^{T} K(t, s) f\left(s, x(s), \int_{0}^{s} g(s, \tau, x(\tau)) d \tau\right) d s+
$$




$$
+\sum_{k=1}^{P} K\left(t, t_{k}\right) I_{k}\left(x\left(t_{k}\right)\right)
$$

for $t \in\left(t_{i}, t_{i+1}\right), \quad i=0,1,2, \ldots, p$.

Obviously, the fixed points of the operator $F$ are the solutions of boundary value problem (2.1) - (2.3). By means of the compressive operators principle we show that the operator $F$ determined by equality (4.2) has a unique fixed point.

Let $x, y \in P C\left([0, T] ; R^{n}\right)$ be any fixed elements. Then for any $t \in\left(t_{i}, t_{i+1}\right]$ we have

$$
\begin{gathered}
|F(x)(t)-F(y)(t)| \leq \\
\leq \int_{0}^{T}|K(t, s)| \cdot\left|f\left(s, x(s), \int_{0}^{s} g(s, \tau, x(\tau)) d \tau\right)-f\left(s, y(s), \int_{0}^{s} g(s, \tau, y(\tau)) d \tau\right)\right| d s+ \\
+\sum_{k=1}^{P}\left|K\left(t_{i}, t_{k}\right)\right| \cdot\left|I_{k}\left(x\left(t_{k}\right)\right)-I_{k}\left(y\left(t_{k}\right)\right)\right| .
\end{gathered}
$$

Using conditions (H1), (H2), from the last inequality we obtain

$$
\begin{gathered}
|F(x)(t)-F(y)(t)| \leq \\
\leq S M_{1} \int_{0}^{T}\left\{|x(t)-y(t)|+\left|\int_{0}^{t} g(t, s, x(s)) d s-\int_{0}^{t} g(t, s, y(s)) d s\right|\right\} d t+ \\
\left.+S \sum_{k=1}^{P} l_{k} \mid x\left(t_{k}\right)\right)-y\left(t_{k}\right) \mid \leq \\
\left.\leq S M_{1}\left\{T\|x-y\|+M_{2} \frac{T^{2}}{2}\|x-y\|\right\}+S \sum_{k=1}^{P} l_{k} \mid x\left(t_{k}\right)\right)-y\left(t_{k}\right) \mid .
\end{gathered}
$$

We can rewrite this inequality in the form

$$
|F(x)(t)-F(y)(t)| \leq\left[S\left(M_{1} T\left(1+\frac{M_{2} T}{2}\right)+\sum_{k=1}^{P} l_{k}\right)\right] \times\|x-y\|_{P C}
$$

Thus,

$$
\|F(x)(t)-F(y)(t)\| \leq L\|x-y\|_{P C} .
$$

Here, taking into account condition (4.1) we obtain that the operator $F$ is compressive. According to the fixed point principle, we can conclude that the operator $F$ has a unique fixed point. This is equivalent to the fact that nonlocal boundary value problem (2.1) - (2.3) has a unique solution.

The theorem is proved.

The second result of the section is devoted to establishing the existence of solutions of boundary value problem (2.1) - (2.3) that is based on Schaefer's fixed point.

Theorem 4.2. Suppose that the following conditions are fulfilled:

(H3) The function $f:[0, T] \times R^{n} \times R^{n} \rightarrow R^{n}$ is continuous and there exists a constant $N_{1}>0$ such that

$$
|f(t, x, y)| \leq N_{1}
$$


for all $t \in[0, T]$ and $(x, y) \in R^{2 n}$;

(H4) The functions $I_{k}: R^{n} \rightarrow R^{n}$ are continuous and there exists a constant $N_{2}>0$ such that

$$
\max _{k \in\{1,2, \ldots, P\}}\left|I_{k}(x)\right| \leq N_{2} .
$$

Then boundary value problem (2.1)-(2.3) has at least one solution on $[0, T]$.

Proof. Show that under the above conditions, the operator $F(x)(t)$ determined by equality (4.2) has fixed points. This will be done after certain steps.

Step 1. Under the conditions of the theorem, the operator $F$ is continuous in $P C\left([0, T] ; R^{n}\right)$. Let $\left\{x_{n}\right\}$ be a functional sequence in space $P C\left([0, T] ; R^{n}\right)$ and $x_{n} \rightarrow x, x \in P C\left([0, T] ; R^{n}\right)$. Then for any $t \in\left[t_{i}, t_{i+1}\right]$, and $i=0,1, \ldots, p$

$$
\begin{gathered}
\left|F\left(x_{n}\right)(t)-F(x)(t)\right| \leq \int_{0}^{T}|K(t, s)| \times \\
\times\left|f\left(s, x_{n}(s), \int_{0}^{s} g\left(s, \tau, x_{n}(\tau)\right) d \tau\right)-f\left(s, x(s), \int_{0}^{s} g(s, \tau, x(\tau)) d \tau\right)\right| d s+ \\
+\sum_{k=1}^{P}\left|K\left(t, t_{k}\right)\right| \cdot\left|I_{k}\left(x_{n}\left(t_{k}\right)\right)-I_{k}\left(x\left(t_{k}\right)\right)\right| .
\end{gathered}
$$

Here taking into account conditions (H3), (H4), we have:

$$
\begin{gathered}
\left|F\left(x_{n}\right)(t)-F(x)(t)\right| \leq \\
\leq S T \max _{s \in[0, T]}\left|f\left(s, x_{n}(s), \int_{0}^{s} g\left(s, \tau, x_{n}(\tau)\right) d \tau\right)-f\left(s, x(s), \int_{0}^{s} g(s, \tau, x(\tau)) d \tau\right)\right|+ \\
+S \sum_{k=1}^{P}\left|I_{k}\left(x_{n}\left(t_{k}\right)\right)-I_{k}\left(x\left(t_{k}\right)\right)\right| .
\end{gathered}
$$

Since the functions $f, g$ and $I_{k}, k=1,2, \ldots, p$, are continuous, we have

$$
\left\|F\left(x_{n}\right)(t)-F(x)(t)\right\|_{P C} \rightarrow 0
$$

as $n \rightarrow \infty$.

Step 2. The mapping $F$ is bounded in space $P C\left([0, T] ; R^{n}\right)$. This is equivalent to the fact that we should show that for any $\eta>0$ there exists $l>0$ such that for any

$$
x \in B_{\eta}=\left\{x \in P C\left([0, T] ; R^{n}\right):\|x\| \leq \eta\right\}
$$

there is

$$
\|F(x(\cdot))\| \leq l .
$$

Applying the triangle inequality and using the assumptions (H3) and (H4), for $t \in\left(t_{i}, t_{i+1}\right]$, we get

$$
\begin{gathered}
|F(x)(t)| \leq \\
\leq \int_{0}^{T}|K(t, s)| \cdot\left|f\left(s, x(s), \int_{0}^{s} g(s, \tau, x(\tau)) d \tau\right)\right| d s+
\end{gathered}
$$




$$
+\sum_{i=1}^{P}\left|K\left(t, t_{i}\right)\right| \cdot\left|I_{i}\left(x\left(t_{i}\right)\right)\right|+\left\|N^{-1} B\right\| .
$$

Thus,

$$
|F(x)(t)| \leq\left\|N^{-1} B\right\|+S\left[T N_{1}+p N_{2}\right]=l .
$$

Step 3. The operator $F$ maps the bounded set into equicontinuous subset of the space $P C\left([0, T], R^{n}\right)$. Let $\tau_{1}, \tau_{2} \in\left(t_{i}, t_{i+1}\right]$ and $\tau_{1}<\tau_{2} . B_{\eta}$ be a bounded set in step 2 and let $x \in B_{\eta}$. Then we have:

$$
\begin{aligned}
& F(x)\left(\tau_{2}\right)-F(x)\left(\tau_{1}\right)= \\
& =N^{-1} \int_{0}^{\tau_{2}}\left(A+\int_{0}^{s} n(\tau) d \tau\right) f\left(s, x(s), \int_{0}^{s} g(s, \tau, x(\tau)) d \tau\right) d s- \\
& -N^{-1} \int_{\tau_{2}}^{T} \int_{s}^{T} n(\tau) d \tau f\left(s, x(s), \int_{0}^{s} g(s, \tau, x(\tau)) d \tau\right) d s- \\
& -N^{-1} \int_{0}^{\tau_{1}}\left(A+\int_{0}^{s} n(\tau) d \tau\right) f\left(s, x(s), \int_{0}^{s} g(s, \tau, x(\tau)) d \tau\right) d s+ \\
& +N^{-1} \int_{\tau_{1}}^{T} \int_{s}^{T} n(\tau) d \tau f\left(s, x(s), \int_{0}^{s} g(s, \tau, x(\tau)) d \tau\right) d s \\
& =N^{-1} \int_{\tau_{1}}^{\tau_{2}}\left(A+\int_{0}^{s} n(\tau) d \tau\right) f\left(s, x(s), \int_{0}^{s} g(s, \tau, x(\tau)) d \tau\right) d s+ \\
& +N^{-1} \int_{\tau_{1}}^{\tau_{2}} \int_{s}^{T} n(\tau) d \tau f\left(s, x(s), \int_{0}^{s} g(s, \tau, x(\tau)) d \tau\right) d s= \\
& =\int_{\tau_{1}}^{\tau_{2}} f\left(s, x(s), \int_{0}^{s} g(s, \tau, x(\tau)) d \tau\right) d s .
\end{aligned}
$$

Hence

$$
\left|F(x)\left(\tau_{1}\right)-F(x)\left(\tau_{2}\right)\right| \leq \int_{\tau_{1}}^{\tau_{2}}\left|f\left(s, x(s), \int_{0}^{s} g(s, \tau, x(\tau)) d \tau\right)\right| d s
$$

As $\tau_{1} \rightarrow \tau_{2}$, the right hand side of the preceding inequality tends to zero. Taking into account that the mapping $F$ is continuous and equivalently continuous, we conclude that the mapping

$$
F: P C\left([0, T], R^{n}\right) \rightarrow P C\left([0, T], R^{n}\right)
$$

is completely continuous.

Step 4. Show that the set

$$
\Delta=\left\{x \in P C\left([0, T], R^{n}\right): x=\lambda F(x)\right\},
$$


for some $0<\lambda<1$ is bounded. Let for some $0<\lambda<1$ the equality $x=\lambda(F x)$ be fulfilled. Then for any $t \in\left(t_{i}, t_{i+1}\right], \quad i=0,1, \ldots, p$, we have

$$
\begin{gathered}
x(t)= \\
=\lambda\left[N^{-1} B+\int_{0}^{T} K(t, s) f\left(s, x(s), \int_{0}^{s} g(s, \tau, x(\tau)) d \tau\right) d s+\sum_{k=1}^{P} K\left(t_{i}, t_{k}\right) I_{n}\left(x\left(t_{k}\right)\right)\right] .
\end{gathered}
$$

Hence, taking into account assumptions (H3) and (H4) (as in step 2) for any $t \in[0, T]$ we have

$$
|F(x)(t)| \leq\left[N_{1} T+p N_{2}\right] S+\left\|N^{-1} B\right\| .
$$

Consequently, we have

$$
\|x\|_{P C} \leq\left\|N^{-1} B\right\|+\left[N_{1} T+p N_{3}\right] S=R .
$$

This shows that the set $\Delta$ is bounded. So, all the conditions of Schauffer's fixed point theorem are fulfilled. Hence it follows that the operator $F$ has fixed points that are the solutions of boundary value problem (2.1)-(2.3).

The theorem is proved.

Now we show continuous dependence of solutions of problem (2.1)-(2.3) on the right hand side of $(2.2)$.

Theorem 4.3. Let conditions (H1), (H2) be fulfilled, and $L<1$. Then for any $B_{1}, B_{2} \in R^{n}$ and for appropriate solutions $x_{1}, x_{2}$ of the following boundary value problems

$$
\begin{gathered}
\dot{x}_{j}(t)=f\left(t, x_{j}(t), \int_{0}^{t} g\left(t, s, x_{j}(s)\right) d s\right), t \in[0, T], t \neq t_{i}, i=1,2, \ldots, p, \\
A x_{j}(0)+\int_{0}^{T} n(t) x_{j}(t) d t=B_{j}, \\
x_{j}\left(t_{i}^{+}\right)-x_{j}\left(t_{i}\right)=I_{i}\left(x_{j}\left(t_{i}\right)\right), \quad i=1,2, \ldots, p, \quad j=1,2,
\end{gathered}
$$

the estimation

$$
\left\|x_{1}(t)-x_{2}(t)\right\| \leq(1-L)^{-1}\left\|N^{-1}\right\|\left\|B_{1}-B_{2}\right\| .
$$

is fulfilled.

Proof. Let $B_{1}, B_{2} \in R^{n}$ be any points, and $x_{1}, x_{2}$ be appropriate solutions of problem (4.3)-(4.5). Then we have:

$$
\begin{gathered}
x_{1}(t)-x_{2}(t)=N^{-1}\left[B_{1}-B_{2}\right]+ \\
+\int_{0}^{T} K(t, s)\left[f\left(s, x_{1}(s), \int_{0}^{s} g\left(s, \tau, x_{1}(\tau)\right) d \tau\right)-f\left(s, x_{2}(s), \int_{0}^{s} g\left(s, \tau, x_{2}(\tau)\right) d \tau\right)\right] d s+ \\
+\sum_{k=1}^{P} K\left(t, t_{k}\right)\left[I_{k}\left(x_{1}\left(t_{k}\right)\right)-I_{k}\left(x_{2}\left(t_{k}\right)\right)\right]
\end{gathered}
$$

Now, using conditions (H1) and (H2), from (4.6) we get

$$
\left|x_{1}(t)-x_{2}(t)\right| \leq\left\|N^{-1}\left[B_{1}-B_{2}\right]\right\|+
$$




$$
\begin{gathered}
+S M_{1} \int_{0}^{T}\left\{\left|x_{1}(\tau)-x_{2}(\tau)\right|+M_{2} \int_{0}^{\tau}\left|x_{1}(s) x_{2}(s) d s\right|\right\} d \tau+ \\
+S \sum_{i=1}^{p} l_{i}\left|x_{1}\left(t_{k}\right)-x_{2}\left(t_{k}\right)\right| .
\end{gathered}
$$

Hence

$$
\begin{gathered}
\left\|x_{1}(t)-x_{2}(t)\right\| \leq\left\|N^{-1}\right\|\left\|B_{1}-B_{2}\right\|+ \\
+S\left(M_{1} T\left(1+\frac{M_{2} T}{2}\right)+\sum_{k=1}^{p} l_{k}\right)\left\|x_{1}(t)-x_{2}(t)\right\| .
\end{gathered}
$$

As $L<1$, from the last inequality it follows that

$$
\left\|x_{1}(t)-x_{2}(t)\right\| \leq(1-L)^{-1}\left\|N^{-1}\right\|\left\|B_{1}-B_{2}\right\| .
$$

The theorem is proved.

Note that the scheme suggested in the paper can be successfully used in more complicated boundary value problems with impulse action. For example, for a boundary value problem when (2.2) contains two-point or multipoint and integral terms.

\section{References}

[1] V.M. Abdullayev, Numerical solution to optimal control problems with multipoint and integral conditions. Proceedings of the Institute of Mathematics and Mechanics, National Academy of Sciences of Azerbaijan, 44 (2018), no. 2, 171-186.

[2] K.R. Aida-zade, An approach for solving nonlinearly loaded problems for linear ordinary differential equations. Proceedings of the Institute of Mathematics and Mechanics, National Academy of Sciences of Azerbaijan, 44 (2018), no. 2, 338350.

[3] A. Anguraj, M. Mallika Arjunan, Existence and uniqueness of mild and classical solutions of impulsive evolution equations, Elect. J. Differential Equations, 2005 (2005), no. 111, 1-8.

[4] A. Ashyralyev, Y.A. Sharifov Optimal Control Problems for Impulsive Systems with Integral Boundary Conditions, EJDE, 2013 (2013), no. 80, 1-11.

[5] A. Ashyralyev, Y.A. Sharifov, Existence and uniqueness of solutions for nonlinear impulsive differential equations with two-point and integral boundary conditions, Advances in difference equations, 2013, 2013:173.

[6] M. Benchohra, J. Henderson, S.K. Ntouyas, Impulsive differential equations and inclusions. Contemporary mathematics and its application, Hindawi Publishing Corporation. New York. 2: 2006.

[7] L. Bin, L. Xinzhi, L. Xiaoxin. Robust global exponential stability of uncertain impulsive systems. Acta Mathematika Scientia, 25 B(1) (2005), 161-169.

[8] A.A. Boichuk, A.M. Samoilenko, Generalized inverse operators and Fredholm boundary-value problems (2nd ed.), Berlin/Boston: Walter de Gruyter GmbH, 2016, $314 \mathrm{pp}$.

[9] A.A. Boichuk, A.M. Samoilenko, Generalized Inverse Operators and Fredholm Boundary-Value Problems, Brill, Utrecht., 2004.

[10] A. Halanay, D. Wexler. Quality theory of impulse systems, Moscow, Mir, 1971, 309 pp. 
[11] Sh. Ji, Sh. Wen, Nonlocal Cauchy Problem for Impulsive Differential Equations in Banach Spaces. International Journal of nonlinear Science, 10 (2010), no. 1, 88-95.

[12] V. Lakshmikantham, D.D. Bainov, P.S. Simeonov, Theory of Impulsive Differential Equations, World Scientific. Singapore, 1989, 434 pp.

[13] M. Li, M. Han, Existence for neutral impulsive functional differential equations with nonlocal conditions. Indagationes Mathematicae, 20 (2009), no. 3, 435-451.

[14] M.J. Mardanov, Y.A. Sharifov, Existence results for first order nonlinear impulsive differential equations with nonlocal boundary conditions, AIP Conference Proceedings 1676, 020015 (2015).

[15] N.A.Perestyk, V.A. Plotnikov, A.M. Samoilenko, N.V. Skripnik, Differential equations with impulse effect: multivalued right-hand sides with discontinuities, DeGruyter Stud. Math. - Berlin: Walter de Gruter Co., 2011, 40 pp.

[16] A.M. Samoilenko, N.A. Perestyuk, Impulsive differential equations, Kiev, Visha Sckola, 1987, $287 \mathrm{pp}$.

[17] A.M. Samoilenko, N. A. Perestyk, Impulsive differential equations, Singapore: World Sci., 1995.

[18] B. Selvaraj, M. Mallika Arjunan, V.Kavitha, Existence of solutions for impulsive nonlinear differential equations with nonlocal conditions, J. KSIAM 13 (2009), no.3, 203-215.

[19] Ya. Sharifov, Optimal control problems for impulsive systems under nonlocal boundary conditions, Vestnik Sam.. Gos. Tekhn. Univ., ser. Phys. math. sci., 33 (2013), no. $4,(33), 34-45$.

[20] Ya. Sharifov, Optimal control for impulsive systems under nonlocal boundary conditions, Russian Mathematics, 57 (2013), no. 2, 65-72.

[21] Y.A. Sharifov, N.B. Mammadova, Optimal control problem described by impulsive differential equations with nonlocal boundary conditions, Differential equations, $\mathbf{5 0}$ (2014), no. 3, 403-411.

[22] Y.A. Sharifov, Conditions Optimality in Problems Control with Systems Impulsive Differential Equations Under Non-Local Boundary Conditions, Ukrainian Mathematical Journal, 64 (2012), no. 6, 836-847.

Misir J. Mardanov

Institute of Mathematics and Mechanics, National Academy of Sciences of Azerbaijan, Az-1141, Baku, Azerbaijan

E-mail address: misirmardanov@yahoo.com

Yagub A. Sharifov

Baku State University, 23, Z.Khalilov str.,, Baku, Azerbaijan

E-mail address: ysharifov@bsu.edu.az

Farah M. Zeynalli

Ganja State University, Ganja, Azerbaijan

E-mail address: farahzeynalli@rambler.ru

Received: June 5, 2019; Accepted: August 21, 2019 